\title{
Информаиионная поддержка принятия решений при возникновении аварийной ситуаиии на объектах газопровода на основе продукционных правил
}

\author{
О.И. Христодуло ${ }^{1}$, д.т.н., професссор, зав. кафедрой геоинформаиионных систем, \\ office@ugatu.su \\ A.C. Самойлов ${ }^{2}$, начальник группь геоинформаиионных систем, оffice@ugatu.su \\ 1 Уфимский государственный авиаиионный технический университет, \\ 2. Уфа, 450008, Россия \\ ${ }^{2}$ Компания "Газпром газораспределение Уфа", г. Уфра, 450099, Россия
}

Обеспечение газом промышленных предприятий осуществляется специализированными организациями с помощью совокупности комплексов иерархически и территориально распределенных объектов, которые взаимосвязаны между собой на региональном уровне, но в то же время являются частью системы обеспечения газом федерального уровня. Процесс обеспечения газом промышленных предприятий связан с обработкой информации об объектах, специфика которой заключается в важной роли пространственной составляющей.

В статье проведен анализ проблемы обеспечения газом промышленных предприятий. Рассмотрены особенности информации и пространственных объектов, использующихся в процессе обеспечения газом. Обоснована необходимость использования геоинформационной системы для поддержания в актуальном состоянии, обработки, анализа, контроля целостности и непротиворечивости информации о пространственных объектах газораспределительной организации. Приведены пространственная информация об объектах обеспечения газом промышленных предприятий (газораспределительные станции, газопроводы высокого и среднего давления, запорная арматура, пункты редуцирования газа, места возможных возникновений аварий и прочее), основные характеристики этих объектов. Кроме того, описаны продукционные правила, составленные на основе формализованных знаний экспертов и описывающие действия диспетчера при возникновении аварийной ситуации на газопроводе.

Представлена схема использования базы знаний на основе продукционных правил для информационной поддержки принятия решений при возникновении аварийной ситуации. Информационная поддержка принятия решений по локализации аварийной ситуации на объектах обеспечения газом промышленных предприятий основывается на использовании пространственной информации об объектах непосредственно из распределенной базы пространственных данных. Анализ данных с помощью формализованных экспертных знаний позволяет моделировать аварийную ситуацию, рассчитывать объем запаса газа в трубопроводе и формировать необходимые отчеты.

Ключевые слова: информационная поддержка, аварийная ситуация, объекты газопровода, пространственная информация, продукционные правила.

Ведущую роль в развитии экономики России и отдельно взятых регионов занимают промышленные предприятия, число которых составляет более 29,9 тысячи объектов. Одним из наиболее важных ресурсов, необходимых для стабильной работы промышленных предприятий, является природный газ, его доля среди прочих энергоресурсов равна 29 \%. Структура потребления энергоресурсов промышленными предприятиями показана на рисунке 1.

Бесперебойное, надежное и безопасное снабжение газом промышленных предприятий регионов выполняется газораспределительными организациями. Они имеют сложную иерархическую структуру, территориально распределены и являются частью системы федерального уровня. Как и любое промышленное оборудование, газопроводы и объекты на них, выполняющие задачу транспортировки газа до конечного промышленного потребителя, требуют обслуживания и поддержания их в работоспособном состоянии.

Как правило, различные подразделения газораспределительных организаций создают информационные системы, содержащие различную и зачастую противоречивую информацию об одних и тех же объектах, поскольку обмен данными (синхронизация) между этими системами не настроен [1]. В силу того, что основные объекты инфраструктуры газоснабжения 


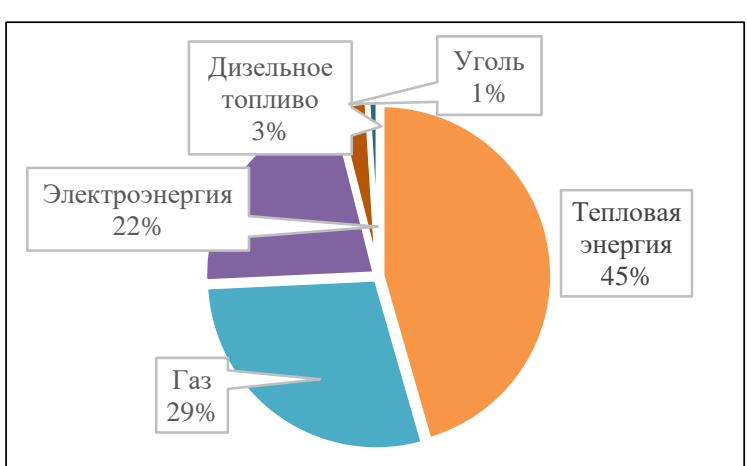

Рис. 1. Структура потребления энергоресурсов промышленными предприятиями России

Fig. 1. The structure of energy consumption by industrial enterprises of the Russian Federation

(газопроводы, пункты редуцирования газа, запорная арматура, изолирующие фланцевые соединения, промышленные потребители и т.д.) имеют пространственную составляющую, актуально использование геоинформациинных систем (ГИС), позволяющих осуществлять сбор, хранение, редактирование, поддержание в актуальном состоянии, обработку, анализ, контроль целостности и непротиворечивости информации о пространственных объектах, а также консолидацию информации и интеграцию с другими информационными системами [2, 3].

Для поддержки принятия решений при возникновении аварийной ситуации на объектах обеспечения газом промышленных предприятий требуется наличие пространственной и атрибутивной информации об обслуживаемых объектах (промышленные предприятия, газораспределительные станции, газопроводы высокого и среднего давления, запорная арматура, пункты редуцирования газа, места возможных аварий и пр.) (рис. 2) [4, 5].

В случае возникновения аварийной ситуации на газопроводе специалисту, принимающему решение, требуется за короткий промежуток времени проанализировать наиболее важные характеристики [6], основными из которых являются следующие.

1. Точное пространственное расположение места возникновения аварийной ситуации на сегменте газопровода. Место возникновения аварии является значимым фактором, поскольку возможны ситуации, когда на небольшом расстоянии друг от друга могут располагаться газопроводы, от которых питаются различные промышленные объекты, что, в свою очередь, обусловливает перекрытие различной запорной арматуры для локализации аварийного участка.

2. Объем запаса газа в газопроводе. Поскольку одним из основных требований для организаций, осуществляющих поставку газа потребителям, является бесперебойность, при возникновении аварийной ситуации и локализации аварийного участка в определенных случаях, при достаточно большом запасе газа в газопроводе возможно в течение некоторого времени обеспечение потребителей газом. Соответственно, расчет запаса газа в отключаемом газопроводе является критичным параметром при планировании аварийно-восстановительных работ [7].

Для принятия эффективных решений в результате анализа этих характеристик предлагается использовать модель представления знаний, которая будет базироваться на продукционных правилах [8].

Газопровод, по которому осуществляется поставка газа промышленным предприятиям $L$, обозначим как совокупность множества координат $L=\left\{\left(X^{L}, Y^{L}\right)\right\}$. Место возникновения аварии $L$ имеет координаты $\left(x^{a}, y^{a}\right)$, пункт редуци-

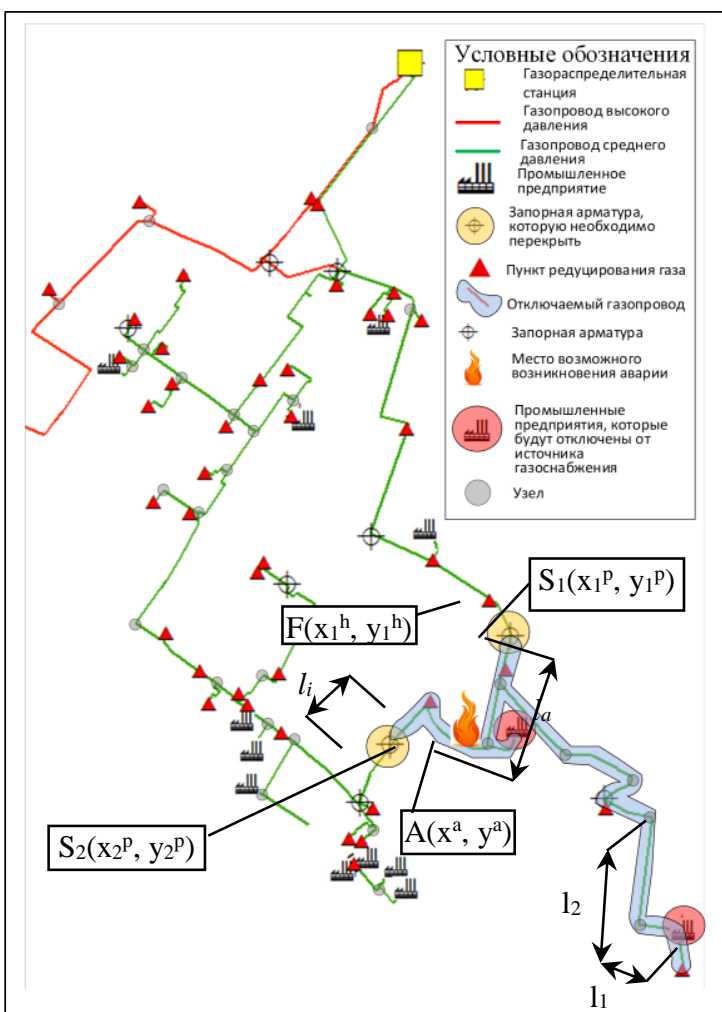

Рис. 2. Пространственная информация об объектах обеспечения газом промышленных предприятий

Fig. 2. Spatial information on gas supply facilities of industrial enterprises 
рования газа $F$ имеет координаты $\left(x^{h}, y^{h}\right)$. Обозначим объем запаса газа на отключаемом сегменте газопровода как функцию $Q_{i}=Q_{i 1}(V) \times$ $\times Q_{i 2}(T, P, z)$, а суммарный фактический расход газа потребителем на отключаемом участке как $W_{c}$, тогда продукционные правила можно формализованно записать в виде таблицы.

На рисунке 3 представлена схема использования разработанной базы знаний для оказания информационной поддержки принятия решения при возникновении аварийной ситуации на объектах поставки газа промышленным предприятиям.

Таким образом, применение предложенных продукционных правил, разработанных на основе формализованных экспертных знаний и являющихся частью базы знаний, позволит провести анализ и выполнить обработку про-

\section{Фрагмент правил базы знаний A fragment of the knowledge base rules}

\begin{tabular}{|c|c|c|}
\hline & Условие выбора & Правила выбора пространственных данных и действия диспетчера \\
\hline$R_{1}$ & \begin{tabular}{|l|} 
ЕСЛИ $x^{a} \subset X^{L}$ \\
И $y^{a} \subset Y^{L}$ \\
И PRESSURE- \\
CLASS = 'низк. IV \\
$(<0,005$ МПа)' \\
И $Q_{i}>W_{c}$ ТО
\end{tabular} & $\begin{array}{l}\text { ЕСЛИ координаты места возникновения аварии совпадают с указанным газопро- } \\
\text { водом И авария на газопроводе низкого давления И запас газа в этом газопроводе } \\
\text { БОЛЬШЕ, чем суммарный часовой расход газа потребителями на отключаемом } \\
\text { участке, ТО 1. Найти и ознакомить с информацией о пространственных объектах, } \\
\text { на которых произошла авария, бригаду. 2. Найти альтернативный источник по- } \\
\text { дачи газа. 3. Укомплектовать мобильную бригаду необходимыми средствами. } \\
\text { 4. Обеспечить выезд бригады в течение } 5 \text { минут на объект. 5. Направить автомо- } \\
\text { биль снабжения со склада с необходимым оборудованием для устранения аварии } \\
\text { на объект. 6. Контроль проведения работ. 7. При необходимости отключения по- } \\
\text { требителей сообщить руководителям промышленных предприятий и отопитель- } \\
\text { ных котельных о прекращении подачи газа до ликвидации аварии на газопро- } \\
\text { воде. 8. Дать команду на отключение газа. 9. Дать команду на пуск газа }\end{array}$ \\
\hline$R_{2}$ & \begin{tabular}{|l|} 
ЕСЛИ $x^{a} \subset X^{L}$ \\
И $y^{a} \subset Y^{L}$ \\
И PRESSURE- \\
CLASS = 'низк. IV \\
$(<0,005$ МПа)' \\
И $Q_{i}=W_{c}$ ТО
\end{tabular} & $\begin{array}{l}\text { ЕСЛИ координаты места возникновения аварии совпадают с указанным газопро- } \\
\text { водом И авария на газопроводе низкого давления И запас газа в этом газопроводе } \\
\text { РАВЕН суммарному часовому расходу газа потребителями на отключаемом } \\
\text { участке, ТО 1. Поиск и ознакомление с информацией о пространственных объек- } \\
\text { тах, на которых произошла авария. 2. Укомплектовать мобильную бригаду необ- } \\
\text { ходимыми средствами. 3. Обеспечить выезд бригады в течение } 5 \text { минут на объ- } \\
\text { ект. 4. Направить автомобиль снабжения со склада с необходимым оборудова- } \\
\text { нием для устранения утечки газа к месту аварии. 5. При необходимости } \\
\text { отключения потребителей сообщить руководителям промышленных предприя- } \\
\text { тий и отопительных котельных о прекращении подачи газа до ликвидации аварии } \\
\text { на газопроводе. 6. Дать команду на отключение газа. 7. Дать команду на пуск газа }\end{array}$ \\
\hline$R_{3}$ & \begin{tabular}{|l|} 
ЕСЛИ $x^{a} \subset X^{L}$ \\
И $y^{a} \subset Y^{L}$ \\
И PRESSURE- \\
CLASS = 'низк. IV \\
$(<0,005$ МПа)' \\
И $Q_{i}<W_{c}$ ТО
\end{tabular} & $\begin{array}{l}\text { ЕСЛИ координаты места возникновения аварии совпадают с указанным газопро- } \\
\text { водом И авария на газопроводе низкого давления И запас газа в этом газопроводе } \\
\text { МЕНЬШЕ суммарного часового расхода газа потребителями на отключаемом } \\
\text { участке, ТО 1. Поиск и ознакомление с информацией о пространственных объек- } \\
\text { тах, на которых произошла авария. 2. Направить на место аварии ближайшую к } \\
\text { месту аварии мобильную бригаду. 3. Направить автомобиль снабжения со склада } \\
\text { с необходимым оборудованием для устранения утечки газа к месту аварии. } \\
\text { 4. Сообщить руководителям промышленных предприятий и отопительных ко- } \\
\text { тельных о прекращении подачи газа до ликвидации аварии на газопроводе. } \\
\text { 5. Дать команду на отключение газа. 6. Дать команду на пуск газа }\end{array}$ \\
\hline$R_{4}$ & $\begin{array}{l}\text { ЕСЛИ } x^{a} \subset X^{L} \\
И y^{a} \subset Y^{L} \\
\text { И (PRESSURE- } \\
\text { СLASS = 'выс. I } \\
(0,6<\text { Р }<1,2 \text { МПа)' } \\
\text { ИЛИ PRESSURE- } \\
\text { CLASS = 'выс. II } \\
(0,3<\text { Р }<0,6 \text { МПа }) \\
\text { И } Q_{i}>W_{c} \text { ТО }\end{array}$ & $\begin{array}{l}\text { ЕСЛИ координаты места возникновения аварии совпадают с указанным газопро- } \\
\text { водом И авария на газопроводе низкого давления И запас газа в этом газопроводе } \\
\text { БОЛЬШЕ, чем суммарный часовой расход газа потребителями на отключаемом } \\
\text { участке, ТО 1. Найти и ознакомить с информацией о пространственных объектах, } \\
\text { на которых произошла авария, бригаду. 2. Найти альтернативный источник по- } \\
\text { дачи газа. 3. Укомплектовать мобильную бригаду необходимыми средствами. } \\
\text { 4. Обеспечить выезд бригады в течение } 5 \text { минут на объект. 5. Направить автомо- } \\
\text { биль снабжения со склада с необходимым оборудованием для устранения аварии } \\
\text { на объект. 6. Контроль проведения работ. 7. При необходимости отключения по- } \\
\text { требителей сообщить руководителям промышленных предприятий и отопитель- } \\
\text { ных котельных о прекращении подачи газа до ликвидации аварии на газопро- } \\
\text { воде. 8. Дать команду на отключение газа. 9. Дать команду на пуск газа }\end{array}$ \\
\hline
\end{tabular}




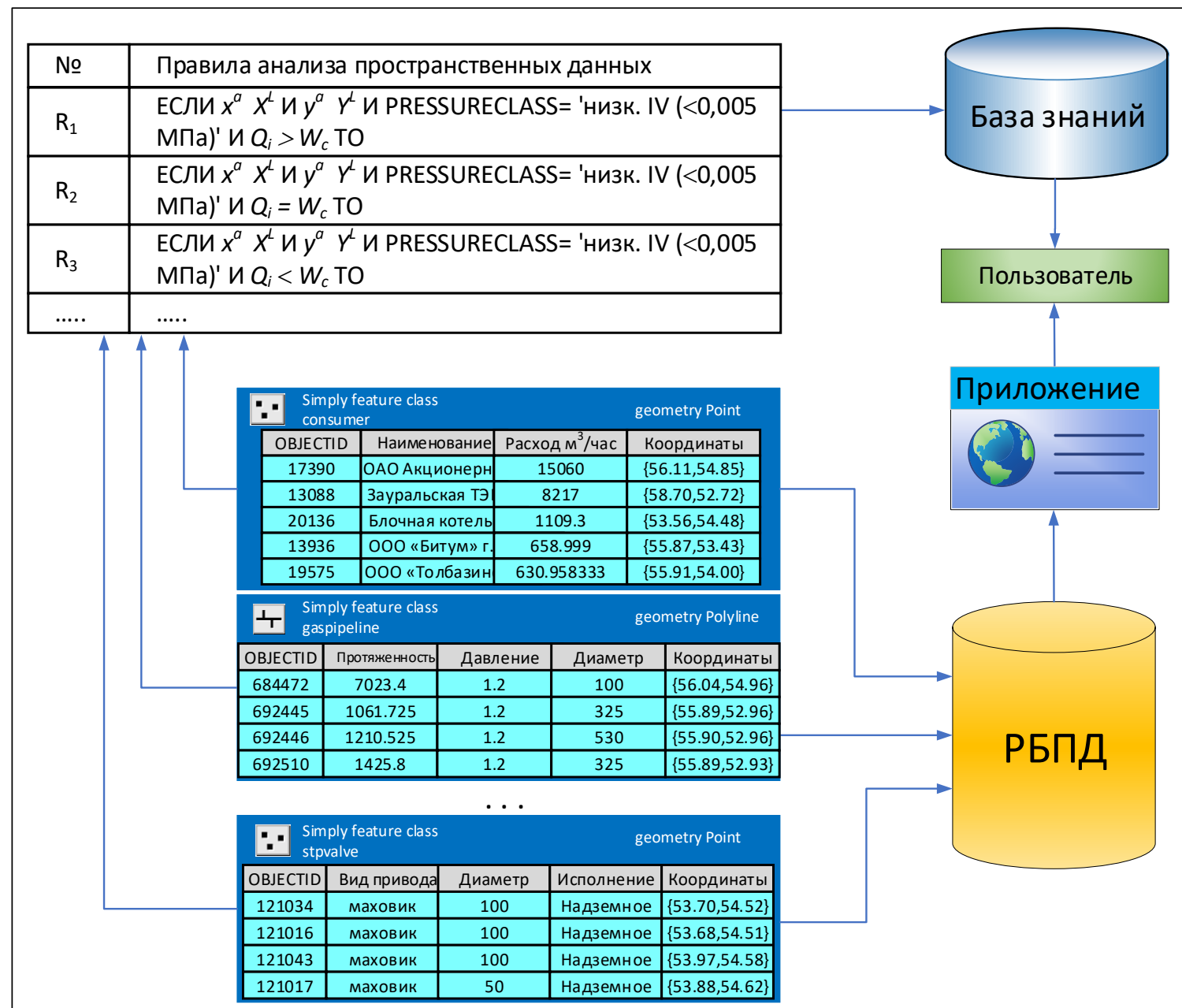

Рис. 3. Схема использования базы знаний для информационной поддержки принятия решений при возникновении аварийной ситуаџии

Fig. 3. The scheme of using the knowledge base for decision-making information support in case of an emergency

странственной информации об объектах обеспечения газом промышленных предприятий, что создаст информационную поддержку принятия решений при возникновении аварийной ситуации [9, 10] и тем самым сократит время для принятия решений в реальном времени, повысит надежность и бесперебойность обеспечения потребителей газом.

\section{Лuтература}

1. Коваленко В.В., Иванченко А.Н. Модель системы информационной поддержки принятия решений в условиях ограниченности ресурсов на крупном промышленном предприятии // Изв. вузов. Сев.-Кавказ. регион. Технич. науки. 2018. № 2. С. 33-39. DOI: 10.17213/0321-2653-2018-2-33-39.

2. Самойлов А.С., Павлов С.В. Проектирование структуры распределенной базы пространственных данных в сложно структурированных иерархических географических информационных системах // Инженер. вестн. Дона. 2015. № 1-1. С 3-8.

3. Черновалова М.В. Алгоритмическое обеспечение информационной системы управления инновационными проектами в промышленности // Программные продукты и системы. 2018. Т. 31 . № 2. С. 330-337.

4. Чуваков А.В. Разработка информационной системы поддержки принятия решений при управлении сложными техническими системами // Символ науки. 2015. № 6. С. 65-72. 
5. Христодуло О.И., Салимзянов И.Ф., Гареева Н.Р. Разработка информационной системы размещения объектов техногенной опасности с использованием нечеткой логики // Науч.-технич. ведомости СПбГПУ. Информатика. Телекоммуникации. Управление. 2015. Т. 5. С. 72-87. DOI: 10.5862/JCSTCS. 229.5 .

6. Башлыков А.А. Анализ подходов к организации интеллектуальной информационной поддержки принятия управляющих решений в нештатных ситуациях // Автоматизация, телемеханизация и связь в нефтяной промышленности. 2014. № 8. С. 3-9.

7. Христодуло О.И., Салимзянов И.Ф., Гареева Н.Р. Разработка многокритериальной математической модели позиционирования объектов техногенной опасности на основе нечеткой логики // Геоинформационные технологии в проектировании и создании корпоративных информационных систем. 2015. C. 55-64.

8. Солдатова О.П. Сравнительный анализ алгоритмов генерации баз нечетких продукционных правил на примере решения задачи классификации // Измерение. Мониторинг. Управление. Контроль. 2014. № 4. С. 43-48.

9. Конобеевских В.В., Мальцев А.С. Использование информационных технологий поддержки принятия решений в чрезвычайных ситуациях // Проблемы обеспечения безопасности при ликвидации последствий чрезвычайных ситуаций. 2015. Т. 2. № 1. С. 53-57.

10. Горемыкина Г.И., Константинова О.В., Мастяева И.Н. Моделирование системы поддержки принятия решений в управлении инвестиционными проектами по предупреждению чрезвычайных ситуаций // Национальные интересы: приоритеты и безопасность. 2017. Т. 13. № 3. С. 514-529.

\title{
Information support for decision-making in the case of an emergency at gas pipeline facilities based on production rules
}

\author{
office@ugatu.su \\ A.S. Samoylov ${ }^{2}$, Head of Group of Geoinformation Systems, office@ugatu.su \\ ${ }^{1}$ Ufa State Aviation Technical University, Ufa, 450008, Russian Federation \\ ${ }^{2}$ Gazprom Gazoraspredeleniye Ufa, Ufa, 450099, Russian Federation
}

O.I. Khristodulo ${ }^{1}$, Dr.Sc. (Engineering), Professor, Head of the Department of Geoinformation Systems,

Abstract. Specialized organizations provide gas to industrial enterprises using a set of complexes of hierarchically and geographically distributed objects that are interconnected at the regional level, but at the same time they are part of the gas providing system at the federal level.

The process of providing gas to industrial enterprises is associated with the processing of information about objects with a special feature consisting in the important role of a spatial component.

The paper analyzes the problem of gas providing to industrial enterprises and considers the features of information and spatial objects used in the process of gas provision. It also demonstrates the need in using the geographic information system to keep up to date, process, analyze, monitor the integrity and consistency of information about spatial objects of a gas distribution organization.

There is a description of the spatial information on gas supply facilities of industrial enterprises (gas distribution stations, high and medium pressure gas pipelines, stop valves, gas reduction points, places of possible accidents, etc.) and the main characteristics of these objects. Moreover, the paper presents production rules based on the formal knowledge of experts and describing the actions of a dispatcher in the case of an emergency on a gas pipeline.

There is a scheme of using a knowledge base based on production rules for information decision support in the case of an emergency. Information support for making decisions on the localization of an emergency at gas supply facilities of industrial enterprises is based on using spatial information about objects directly from a distributed spatial database. Data analysis using formalized expert knowledge allows simulating an emergency, calculating the amount of gas in the pipeline and generating necessary reports.

Keywords: informational support, emergency situation, gas pipeline facilities, spatial information, production rules. 


\section{References}

1. Kovalenko V.V., Ivanchenko A.N. Information decision support system model in conditions of limited resources at a large industrial enterprise. Univ. News. North-Caucas. Reg. Technical Sciences Series. 2018, no. 2, pp. 33-39 (in Russ.). DOI: 10.17213/0321-2653-2018-2-33-39.

2. Samoylov A.S., Pavlov S.V. Designing the structure of a distributed spatial database in complexly structured hierarchical geographic information systems. Engineering J. of Don. 2015, no. 1-1, pp. 3-8 (in Russ.).

3. Chernovalova M.V. Algorithmic support of the information system for managing innovation projects in an industry. Software \& Systems. 2018, no. 2, pp. 330-337 (in Russ.).

4. Chuvakov A.V. Development of a decision-making information system when managing complex technical systems. Science Symbol. 2015, no. 6, pp. 65-72 (in Russ.).

5. Khristodulo O.I., Salimzyanov I.F., Gareeva N.R. The Development of an Information System for Positioning Technogenic Hazard Objects Using Fuzzy Logic. St. Petersburg State Polytech. Univ. J. Computer Science. Telecommunication and Control Systems. 2015, vol. 5, pp. $72-87$ (in Russ.). DOI: 10.5862/JCSTCS. 229.5.

6. Bashlykov A.A. The analysis of approaches to the organization of intellectual information support of operating decisions making in emergency situations. Automation, Telemechanization and Communication in Oil Industry. 2014, no. 8, pp. 3-9 (in Russ.).

7. Khristodulo O.I., Salimzyanov I.F., Gareeva N.R. Development of a multi-criteria mathematical model for positioning man-made hazard objects based on fuzzy logic. Geo-Information Technologies in the Design and Creation of Corporate Information Systems. 2015, pp. 55-64 (in Russ.).

8. Soldatova O.P. Comparative analysis of algorithms of fuzzy production rules' database generation with an example of solving the classification problem. Measuring. Monitoring. Management. Control. 2014, no. 4, pp. 43-48 (in Russ.).

9. Konobeevskikh V.V., Maltsev A.S. The use of information technology decision-making in emergency situations. Security Problems in the Emergency Recovery. 2015, vol. 2, no. 1, pp. $53-57$ (in Russ.).

10. Goremykina G.I., Konstantinova O.V., Mastyaeva I.N. Modeling a decision support system in managing investment projects to prevent emergency situations. National Interests: Priorities and Safety. 2017, vol. 13 , no. 3, pp. 514-529 (in Russ.).

\section{Для цитирования}

Христодуло О.И., Самойлов А.С. Информационная поддержка принятия решений при возникновении аварийной ситуации на объектах газопровода на основе продукционных правил // Программные продукты и системы. 2019. Т. 32. № 3. С. 472-477. DOI: 10.15827/0236235X.127.472-477.

\section{For citation}

Khristodulo O.I., Samoylov A.S. Information support for decision-making in the case of an emergency at gas pipeline facilities based on production rules. Software \& Systems. 2019, vol. 32, no. 3, pp. 472-477 (in Russ.). DOI: 10.15827/0236-235X.127.472-477. 